\title{
Le détachement initial en estonien parlé
}

Son rôle dans la construction et la structuration du discours

Initial detachments in spoken Estonian: their role in the construction and structuration of the discourse

Algteemat sisaldavad lahktarindid suulises eesti keeles: nende roll diskursuse ülesehituses ja struktureerimises

\section{Marri Amon}

\section{OpenEdition \\ Journals}

Édition électronique

URL : https://journals.openedition.org/efo/5258

DOI : 10.4000/efo.5258

ISSN : 2275-1947

Éditeur

INALCO

Édition imprimée

ISBN : 978-2-343-08571-5

ISSN : 0071-2051

Référence électronique

Marri Amon, «Le détachement initial en estonien parlé », Études finno-ougriennes [En ligne], 47 | 2015, mis en ligne le 29 juin 2016, consulté le 20 septembre 2021. URL : http://journals.openedition.org/efo/ 5258 ; DOI : https://doi.org/10.4000/efo.5258

Ce document a été généré automatiquement le 20 septembre 2021.

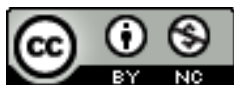

Études finno-ougriennes est mis à disposition selon les termes de la Licence Creative Commons Attribution - Pas d'Utilisation Commerciale 4.0 International. 


\title{
Le détachement initial en estonien parlé
}

\author{
Son rôle dans la construction et la structuration du discours \\ Initial detachments in spoken Estonian: their role in the construction and \\ structuration of the discourse \\ Algteemat sisaldavad lahktarindid suulises eesti keeles: nende roll diskursuse \\ ülesehituses ja struktureerimises
}

\section{Marri Amon}

\section{Introduction}

Cet article analyse le rôle du détachement initial dans la construction et la structuration du discours, en partant d'exemples de l'estonien oral provenant de différents corpus, complétés par quelques exemples de textes écrits. L'analyse se situe dans le cadre de la "structuration informationnelle » qui distingue, d'après l'école de Prague et d'autres auteurs (Enkvist 1984, 1987, Combettes 1983, 1998, Fernandez-Vest 2004, 2006, 2009), en plus des niveaux morphosyntaxique et sémantique, un niveau spécifique de la structuration de l'information. Dans ce cadre, les notions suivantes seront employées : thème, c'est-à-dire ce dont on parle (et dont l'une des formulations sous forme de détachement initial constitue l'objet principal de cet article), rhème, c'est-à-dire ce que l'on dit sur le thème. Cet angle d'approche a été choisi pour les raisons suivantes : d'abord, une étude au niveau de la structuration informationnelle permet d'observer un certain nombre de phénomènes qui ne sont pas discutés en profondeur dans les ouvrages de grammaire ou dans les études sur les textes écrits ; par ailleurs, la structuration de l'information est généralement plus facile à observer dans les textes écrits, tandis que dans la langue orale ces mécanismes se révèlent nettement plus complexes. Puisque la construction qui est au centre de la présente analyse relève avant tout de l'usage oral de la langue, et qu'elle est même considérée par certains auteurs comme un universel dans la langue spontanée, cette étude se concentrera sur 
différents phénomènes intervenant dans la communication réelle, tels que les moyens utilisés pour introduire et pour maintenir les référents dans le discours, leur statut informationnel, l'usage des particules énonciatives pour marquer les constituants informationnels, etc.

2 Cette analyse a pour objectif de mieux comprendre le fonctionnement discursif du détachement initial en estonien parlé ; pour ce faire, les exemples du corpus seront répartis en groupes qui permettent de mieux cerner les caractéristiques essentielles de ces constructions quand il s'agit de les décrire dans leur fonctionnement discursif, à savoir leur structure interne, leur rôle dans construction du discours et la gestion de la référence et dans la structuration des séquences plus longues.

Du point de vue formel, les constructions qui contiennent un élément détaché suivi de la proposition principale ont les caractéristiques suivantes: l'élément détaché est constitué d'un groupe nominal au nominatif (les noms propres sont aussi relativement fréquents comme dans l'exemple 1) qui est repris dans la proposition principale par un pronom coréférentiel; ce dernier porte la marque casuelle appropriée (partitif dans l'exemple ci-dessous). Dans cet exemple, la locutrice Ke pose une question à propos du paiement pour le cours particulier qu'elle vient de prendre, en sachant que l'autre élève, Miku, qui devait participer également n'était pas présent ce jour-là :

\begin{tabular}{|l|l|l|l|l|l|}
\hline$(1)$ & Ke: & aga & kuidas & 'Mikuga & on. \\
\hline & & mais & comment & Miku.CoM & être.3 $\mathrm{sg}^{1}$ \\
\hline
\end{tabular}

\begin{tabular}{|l|l|l|l|l|l|l|l|}
\hline & Kr: & noh' & Miku, & täna & teda & näiteks & 'küll \\
\hline & & PRTCL & Miku & aujourd'hui & he.PART & exemple.TRL & PRTCL \\
\hline
\end{tabular}

\begin{tabular}{|l|l|l|l|l|l|l|}
\hline & ei & olnud & ja nii edasi, & Noh & 'vaata & ise. \\
\hline & & ettre.NEG.PRET. & 3sg et_cetera & PRTCL & regarder.IMP.2sg & même \\
\hline
\end{tabular}

Ke : « Et comment faire avec Miku?»

$\mathrm{Kr}$ : «Bon, Miku, aujourd'hui par exemple il n'était pas là, et cetera, bon, tu n'as qu'à voir toi-même. »

À propos du statut informationnel de ces éléments il convient de préciser que si la proposition principale véhicule dans sa globalité l'information nouvelle, c'est-à-dire l'information qui est nouvelle par rapport au référent précis, dans le contexte précis, le pronom coréférentiel dans cette proposition réfère à l'élément qui est déjà introduit dans la construction détachée (et peut-être a été mentionné auparavant) et qui ne peut donc pas être qualifié de nouveau. Dans différentes analyses, le statut informationnel du référent introduit par la construction détachée n'a pas été défini de manière tout à fait similaire selon les langues étudiées : par exemple, R. Geluykens avance à propos des exemples provenant d'un corpus parlé anglais que les constructions à détachement servent à introduire surtout des référents nouveaux (irrecoverable) - les hésitations ou les connecteurs introductifs tels que par exemple, etc. qui souvent les précèdent, indiquent qu'il s'agit d'une entité dont le statut informationnel nécessite des éléments 
supplémentaires pour être interprétée de manière appropriée (Geluykens 1992, p. 60). En revanche, W.J. Ashby trouve que seulement la moitié des exemples français étudiés dans son analyse comportaient un référent nouveau (Ashby 1988, p. 212); il soutient l'idée selon laquelle la construction avec un groupe nominal détaché est employée avant tout pour changer de thème/topic (topic-shifting, topic-creating) et structurer le discours (ibid., p. 216). C'est une caractéristique qui est avancée dans de nombreuses études, par exemple dans Lambrecht 1994, Gundel 2012.

\subsection{Les constructions à détachement en tant qu'universaux dans la langue spontanée}

Les constructions où un groupe nominal détaché précède la proposition principale qui contient un pronom coréférentiel entrent dans le cadre des études typologiques surtout après la description en 1976 des «topic-prominent languages » par Li \& Thompson; J. Gundel avance en 1988 que ce type de structure est probablement une propriété universelle du discours "non planifié » (Gundel 1988, p. 238-239) et Maslova \& Bernini estiment de même que ces constructions existent dans le registre informel dans toutes les langues, mais que leur degré de grammaticalisation et, partant, leur fréquence varient dans les langues (Maslova \& Bernini 2006, p. 74). Le français est par exemple caractérisé par un usage très fréquent de constructions à détachement, tandis qu'en estonien cette structure, quoique relativement fréquente dans l'oral spontané, reste plus marquée dans les textes écrits et ne constitue pas le moyen le plus typique pour introduire un thème. Cependant, ces constructions semblent assez productives en estonien parlé, ce qui permet par conséquent de tenter une analyse de leurs fonctions dans le discours oral. Mise à part la langue orale (spontanée), il existe d'autres types de textes, proches de la langue orale dans lesquels ces structures sont courantes, tels que les discussions sur Internet, les commentaires des internautes, etc. Dans les textes journalistiques, typiquement, les constructions détachées semblent fréquentes dans les cas où l'on rapporte des paroles d'une personne - on peut parler ici d'une «simulation de l'oral». Dans d'autres cas, en revanche, lorsqu'une construction détachée est employée dans un texte littéraire, par exemple, on peut se demander s'il s'agit surtout de mettre en valeur les caractéristiques de "l'oralité ", comme cela a été parfois suggéré ou bien est-il possible d'identifier d'autres fonctions qui entrent en jeu et qui ne sont pas liées à l'usage « oral » de la langue.

6 En effet, si l'on constate que cette construction est surtout usitée à l'oral, il semble plausible d'en déduire que son emploi à l'écrit sert à mettre en avant l'oralité de l'énoncé en question. Or, quand on analyse les occurrences concrètes, il apparaît que les énoncés (ou les extraits entiers) en question n'ont aucune autre caractéristique de l'oral, il est donc peu probable que ce soit la fonction première de cette structure :

\begin{tabular}{|l|l|l|l|l|l|}
\hline$(2)$ & Kes oli & see, & kes raius & tal & maha pea \\
\hline & quiêtre-PRET & DEM & quicouper-PRET.3sg & il-ADE & ADVtête-GEN \\
\hline
\end{tabular}

\begin{tabular}{|l|l|l|l|l|l|}
\hline ja toppis & suhu & mündi, & Gotlandi & vana & ortugi. \\
\hline
\end{tabular}




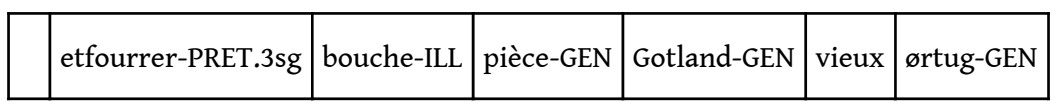

\begin{tabular}{|l|l|l|l|l|l|l|l|l|}
\hline & See & Gotlandi & vana & münt, & see & ei & andnud & mulle \\
\hline & DEM & Gotland-GEN & vieux & pièce & DEM & NEG & donner-NEG.PRET.3sg & je-ALL \\
\hline
\end{tabular}

\begin{tabular}{|l|l|l|}
\hline & hetkekski & asu. \\
\hline moment-TRL + clitique & tranquillité \\
\hline
\end{tabular}

«Qui lui a coupé la tête et lui a fourré dans la bouche une pièce de monnaie, un vieil ørtug de Gotland ? Cette vieille pièce ne m'a pas laissé un instant de repos. »

(I. Hargla Oleviste mõistatus, traduction en français par J-P. Ollivry)

Il s'agit d'un extrait monologique où le personnage principal du roman donne le dénouement de l'histoire. La traduction de l'extrait provient de la traduction publiée du roman et il est intéressant de noter que le traducteur a choisi une plus grande intégration lexicale des éléments, par rapport au texte original (le premier énoncé commençant par «qui était celui qui... » et dans le deuxième énoncé, dans l'original, il y a un détachement initial qui n'est pas repris dans la traduction). Ici, il convient d'envisager les effets stylistiques de ces constructions qui créent une sorte de redondance ou répétition qui peut être interprétée comme un parallèle figuratif à ce long processus de réflexion qui a eu lieu durant tout le récit. Ce constat peut être rapproché de l'idée de McLaughlin qui, en s'appuyant sur les suggestions de Marnette (2005, p. 50-63) souligne le fait que dans les textes littéraires les constructions détachées s'emploient souvent dans les extraits qui présentent le discours intérieur du narrateur (McLaughlin 2011, p. 226). L'absence du point d'interrogation à la fin de la première phrase en estonien étaie cette interprétation, compte tenu du fait que du point de vue lexical et grammatical, il s'agit d'une phrase interrogative.

8 La grammaire estonienne (Erelt et al. 1993) ne fournit pas d'informations sur la fréquence ni sur le degré de grammaticalisation des constructions détachées; elle précise qu'il s'agit de structures (eelteema "pré-thème» et täpsustusjätk «suite spécifiante ») qui ne sont pas liées à la proposition principale par la syntaxe. Le détachement initial, eelteema, est défini comme un constituant de phrase référant à l'élément sur lequel porte le message dans la proposition principale :

\begin{tabular}{|l|l|l|l|l|l|}
\hline (3) & Poiss & -temaga & onmeil & palju & muret. \\
\hline & Garçon & il-COM est & nous-ADE & beaucoup souci- & PART \\
\hline
\end{tabular}

« Le garçon, il nous cause beaucoup de soucis. »

9 La plus récente grammaire descriptive du finnois (Hakulinen et al.2004, p. 972-974, p. 1013-1016) accorde une place importante aux constructions détachées dans l'organisation du discours en finnois, en soutenant que ces structures sont grammaticalisées dans la langue orale spontanée et en décrivant le lien entre les deux constituants (le détachement et la proposition principale) comme étant plutôt de 
nature textuelle et non pas syntaxique. Plusieurs études portant sur le finnois ont mis en avant les fonctions des constructions détachées (Etelämäki 2006, p. 72-82, Helasvuo 2001, p. 126). M.-L. Helasvuo suggère dans son analyse que les constructions à détachement servent en finnois à focaliser sur un référent spécifique (généralement mentionné avant ou extrait d'un ensemble).

Dans la langue orale, les constructions détachées ont entre autres pour fonction de segmenter le discours, de présenter l'information par portions : il sera montré plus loin que les caractéristiques de ces constructions (présentation par un élément au nominatif) permettent d'introduire des référents avec différents statuts informationnels (référents présents dans le discours, référents mentionnés il y a longtemps et réintroduits, référents nouveaux), mais quand il s'agit de textes écrits, cette construction peut sembler redondante. Comme il a été suggéré dans l'analyse de l'exemple (1), elles peuvent avoir des fonctions sur le plan stylistique ou rhétorique, mais c'est une voie qui n'a pas été souvent explorée dans les études linguistiques.

Les constructions avec un détachement initial sont traditionnellement considérées comme un moyen d'introduire le thème ou de marquer la division des constituants d'une phrase en une partie thématique et une partie rhématique (Bally 1944, p. 36). Cependant, tous les détachements ne représentent pas le thème au sens de ce dont on parle : il existe d'autres éléments détachés qui constituent par exemple un cadre spatial ou temporel de l'énoncé ou qui en tant qu'apposition comportent une prédication seconde et par conséquent ne font pas partie des constructions détachées du type qui nous intéresse ici, qui doivent en principe avoir un élément lexical détaché, suivi d'un pronom coréférentiel dans la proposition principale (Combettes 1998, p. 14, 22-24).

Dans cet article, seuls les détachements initiaux seront traités; les détachements finaux, qui, à première vue, pourraient être considérés comme une structure parallèle ayant des fonctions similaires, ont en réalité un certain nombre de caractéristiques et de fonctions qui leur sont propres et ne seront pas abordés dans le cadre de cette analyse.

\subsection{Le détachement initial et le constituant informationnel thème}

13 Le détachement initial a un rapport très clair avec la division de l'énoncé en parties thématique et rhématique. Alors que, dans le cas du détachement initial, il s'agit d'un moyen d'introduire un thème dans le discours, ce n'est à l'évidence pas le seul moyen de le faire : c'est justement là que réside la différence entre les langues qui utilisent peut-être toutes cette structure, mais où le degré de grammaticalisation de ce constituant est assez variable. Quant à l'estonien, sur lequel nous ne disposons pas d'études quantitatives, il est possible d'avancer qu'il ne s'agit pas d'un moyen privilégié d'introduire un thème; en revanche, dans la langue spontanée et dans certains types de textes écrits (qui se rapprochent davantage de la langue parlée, telles les discussions sur Internet, les textes journalistiques, etc.) les détachements initiaux sont utilisés assez fréquemment. De manière générale, les termes ayant un rapport avec la structuration informationnelle, tels que thème, topique, etc, ont été traités à des niveaux très différents (grammaire, sémantique, syntaxe, pragmatique etc.), car les phénomènes linguistiques observés peuvent être analysés à tous ces niveaux et par conséquent il a été proposé de nombreuses définitions, certaines également contradictoires, de sorte 
que nous disposons aujourd'hui de notions qui ne peuvent pas être comprises de manière univoque sans une mise au point préalable.

La notion de thème est comprise ici comme "ce dont parle l'énoncé suivant ». Cette définition qui peut sembler quelque peu intuitive, a été retenue compte tenu de l'objet d'étude clairement délimité, car les autres éléments qui peuvent également être détachés comme les différents cadres (énonciatifs, temporels) et les adverbes, ne sont pas considérés comme faisant partie du type de construction qui est analysé ici.

Il sera question du statut informationnel des référents, mais nous n'allons pas tenter de leur attribuer un statut exact sur une échelle, comme l'ont fait Prince 1981 ou Gundel, Hedberg et Zacharski 1993; nous nous intéresserons davantage aux aspects qui relèvent de la gestion de la référence et des référents dans le discours, à savoir leur présence dans le discours, dans le cadre référentiel (même si la définition et l'interprétation de cette notion n'est pas univoque) et leur persistance dans la conversation.

\section{Le détachement initial dans le corpus}

Dans ce qui suit nous allons étudier des exemples provenant du corpus d'oral spontané, qui vont permettre d'observer les différents aspects liés à l'organisation et la construction du discours à travers le rôle joué par les détachements initiaux. Le corpus est constitué de 115 exemples qui peuvent être répartis en trois groupes à peu près égaux en fonction de leurs caractéristiques formelles : premièrement, les constructions détachées dans les phrases interrogatives, deuxièmement, les éléments détachés modifiés par une proposition relative, et enfin les exemples qui ne font pas partie des deux premiers groupes. Cependant, l'examen des exemples se fera ici selon des critères plus précis qui tiennent compte des aspects liés à la gestion des référents dans le discours et aux caractéristiques internes des éléments détachés. Ces critères nous permettent de créer trois groupes d'exemples :

1. Ajustement référentiel et structure interne de l'élément détaché en tête ;

2. Gestion de la référence dans un contexte avec plusieurs référents concurrents ;

3. Structuration du discours.

18 Ces groupes sont relativement inégaux, car les critères de classement ne relèvent pas du même niveau et la fréquence de ces constructions est prise en considération seulement dans les limites du corpus utilisé, mais ce choix a été fait afin de pouvoir illustrer plus en détail les différentes caractéristiques de ces constructions.

Dans ce qui suit seront discutés les exemples qui représentent chaque groupe défini précédemment.

\subsection{Ajustement référentiel et structure interne de l'élément détaché en tête}

20 Ce groupe comporte des exemples qui possèdent une caractéristique générale qui peut être élargie à la totalité des cas de figure présentés, à savoir la spécification ou l'illustration de la référence, par la focalisation sur un référent ou un groupe de référents. Le plus souvent il s'agit de référents qui sont présents soit dans le discours soit dans le cadre référentiel, mais il peut y avoir aussi des référents introduits 
occasionnellement, sans être mentionnés dans le discours ou identifiables par les autres participants (nouveaux référents). Les exemples ont été sélectionnés afin de pouvoir mettre l'accent sur quelques aspects spécifiques de l'ajustement référentiel. L'énoncé peut par exemple comporter un ajustement référentiel qui concerne l'accord grammatical : dans le discours créé en temps réel, la référence est ajustée de manière continue et le détachement initial permet de le faire par des moyens relativement peu coûteux du point de vue de la charge informationnelle (p.ex. présentation au nominatif des référents longs et complexes).

21 Le détachement initial permet de présenter plusieurs éléments dans une énumération, suivie du rhème qui comporte la reprise pronominale par le pronom need:

\begin{tabular}{|l|l|l|l|l|l|l|}
\hline (4) & P: & aiad & olidküll & lukus & eksole & viljaait \\
\hline & & grange.pl & être-PRET.3plPRTCL & fermé & PRTCL & grange_de_blé \\
\hline
\end{tabular}

\begin{tabular}{|l|l|l|l|l|l|}
\hline & lakaait & kalaait & need & olid & kõiklukus \\
\hline & grange_de_foin & réserve_de_poissons & DEM-pl & être-PRET.3pl & tousfermé \\
\hline
\end{tabular}

« les granges étaient cependant fermées, n'est-ce pas, la grange de blé, la grange de foin, la réserve de poissons, elles étaient toutes fermées à clé »

Cet exemple montre un énoncé qui intervient dans un extrait narratif, avec un sens concessif : le locuteur P explique qu'autrefois il n'y avait pas de criminalité ni de vols dans son village, mais que les différentes dépendances servant au stockage étaient cependant fermées à clé. Le premier énoncé a comme sujet un nom simple au pluriel, aiad "granges", et se termine par la particule eks ole "n'est-ce pas» qui a pour fonction de rapprocher les positions des interlocuteurs et réfère aussi à leurs connaissances communes (les locuteurs sont originaires de la même île). Ensuite, différents types de bâtiments sont spécifiés dans une construction détachée, suivie de la répétition presque à l'identique de la prédication précédente où le quantifieur kõik (« tous ») vient renforcer le pronom de reprise need (« ceux-là »).

La séquence a une structure quelque peu répétitive, compte tenu de l'énumération et d'autres éléments répétitifs (lukus) qui servent à ponctuer l'argumentation du locuteur qui développe son idée en s'appuyant sur plusieurs exemples. Le détachement initial se présente comme une illustration ou une spécification et par sa forme, d'une part, il semble contribuer à la présentation plus structurée de l'information et d'autre part, il permet de marquer un rapport avec le premier énoncé par l'utilisation d'un pronom pluriel qui réfère avant tout à trois éléments qui le précèdent, mais établit également un lien de coréférence aussi le substantif général au pluriel introduit au tout début (aiad « les granges»).

Les référents des éléments détachés entrent dans le cadre général du premier énoncé et constituent des exemples ponctuels, plus spécifiques, qui permettent de soutenir l'argumentation du locuteur. Les référents en question sont introduits pour la première fois dans la discussion et ne seront plus mentionnés ultérieurement.

L'exemple suivant (5) provient d'une conversation téléphonique entre un agent de voyages et sa cliente; le groupe nominal détaché en tête d'énoncé contient une 
subordonnée relative spécifiante qui est relativement longue et comporte plusieurs éléments :

\begin{tabular}{|l|l|l|l|l|l|}
\hline$(5)$ & & 1V:.hhhhhvot & seeet & kuiteil & näiteks\{-\}eeehhhhhh \\
\hline & $(0.4)$ & PRTCLDEMque & sivous- & ADEexemple- & TRL \\
\hline
\end{tabular}

\begin{tabular}{|c|c|c|}
\hline 2 & 'haigestute & ägedalt, \\
\hline & tomber_malade. $2 \mathrm{pl}$ & sérieusement \\
\hline
\end{tabular}

\begin{tabular}{|l|l|l|}
\hline & $3 \mathrm{H}:$ & jah=m \\
\hline & & oui \\
\hline & $(1.1)$ & \\
\hline
\end{tabular}

\begin{tabular}{|l|l|l|l|l|l|l|}
\hline & 4 & $\mathrm{~V}:$ & nii.hhhhhhhh & ja vajate & 'arsti & abi, \\
\hline & & & PRTCL & et avoir_besoin.2pl & médecin-GEN & aide-PART \\
\hline & $(0.3)$ & & & & \\
\hline
\end{tabular}

$$
5 \mathrm{H}: \mathrm{mhmh}
$$

\begin{tabular}{|l|l|l|l|l|l|}
\hline & 6 & V:. hh need & 'kulud & misteil & 'lähevad ütleme teie \\
\hline & DEM.pl & dépense.pl & quevous-ADE & aller-3pldire-1plvous-GEN \\
\hline
\end{tabular}

\begin{tabular}{|l|l|l|l|l|}
\hline & 7 & 'ravimiseks & jate-ae:t & 'ravimiteks \\
\hline & & traitement-TRL & et vous & médicament.pl-TRL \\
\hline
\end{tabular}

\begin{tabular}{|l|l|l|l|l|l|l|}
\hline & 8 & ja 'haiglaraviks & võib-olla & ja=ja & võibolla & arsti \\
\hline & et hospitalisation-TRL & peut-être & etet & peut-être & médecin-GEN \\
\hline
\end{tabular}

\begin{tabular}{|c|c|c|c|c|}
\hline 9 & vi'siidi & tasuks & ja vot & Need 'kaetakse. \\
\hline & visite.GEN & honoraire-TRL & et PRTCL & DEM.plcouvrir-IMPS \\
\hline
\end{tabular}

« ces dépenses que vous aurez disons pour vous soigner et pour vos médicaments et pour l'hospitalisation peut-être et peut-être pour payer les honoraires de la visite médicale, eh bien elles seront remboursées. " 
Il s'agit au départ d'un énoncé conditionnel introduit par kui «si », qui subit une réparation (kui teil näiteks ... haigestute ägedalt) qui est ensuite entrecoupé deux fois par des signaux de rétroaction de la part du locuteur $\mathrm{H}$.

Dans cet exemple on peut relever la particule ja vot qui se place en tant que marqueur de transition entre la subordonnée relative et la proposition principale: sans cet élément la transition serait trop abrupte, compte tenu du fait que le nom tête que complète la relative (need kulud " ces dépenses ») se trouve relativement loin (ligne 6). Le premier élément de la particule ja peut être associé au connecteur ja, employé à plusieurs reprises dans les énumérations qui précèdent. L'énoncé contenant de nombreuses hésitations et réparations, la particule ja vot à la ligne 9 marque la fin de cette séquence et la transition vers la partie rhématique composé du pronom de reprise need « elles » et du verbe à la forme impersonnelle kaetakse « sont couvertes".

Le référent nommé par l'élément détaché est mentionné ici ponctuellement comme une illustration et ne sera pas développé plus loin.

Dans l'exemple suivant (6) il est question d'un même référent général durant toute la séquence (des élèves estoniens et lettons partant en voyage d'études), mais dans la construction détachée un sous-groupe est désigné en utilisant le pronom meie «nos » qui fait référence aux élèves venant d'Estonie et qui permet à la locutrice d'illustrer l'idée que les élèves de cette tranche d'âge ne parlent pas russe, ce qui fait qu'ils sont obligés de parler anglais avec les élèves de Lettonie :

\begin{tabular}{|l|l|l|l|l|l|l|l|}
\hline (6) & 1V: & 'mhmh..hhhh & 'et=äää, & 'et 'nad 'oma'vahel & 'nagu & 'ka & 'siis \\
\hline & & & que & que ils parmi_eux-mêmes & comme & aussi & alors \\
\hline
\end{tabular}

\begin{tabular}{|l|l|l|l|l|l|}
\hline & 2 & 'ika (0.3) mmmmmm (0.6) & 'üldiselt & 'nagu 'meie, & (.)‘ned'ütleme \\
\hline & PRTCL & généralement & commenos & DEM dire-1pl \\
\hline
\end{tabular}

\begin{tabular}{|l|l|l|l|l|l|}
\hline & 3 & neljateist & 'viieteist & 'aastased 'nad & 'vene 'keelt \\
\hline & quatorze & quinze & âgés & ils russe langue.PART \\
\hline
\end{tabular}

\begin{tabular}{|c|c|c|c|c|}
\hline 4 & ‘ei ‘räägi, & ja 'siis' & nad 'omavahel & 'ikkagi \\
\hline & NEGparler-NEG.3pl & et alors ils & parmi_eux-mêmes & quand_même \\
\hline
\end{tabular}

\begin{tabular}{|l|l|l|l|l|l|l|}
\hline & 5 & 'on & 'sunnitud & 'siis ‘rääkima::::(.) & 'inglise \$ & 'keeles, .hhh 'et \\
\hline & & être-AUX.3pl & obliger-PPT & alors parler-SUP & anglais & langue-INE que \\
\hline
\end{tabular}

\begin{tabular}{|c|c|c|c|}
\hline 6 & ‘vähemalt & 'niigi' & palju \\
\hline & au_moins & autant & beaucoup \\
\hline
\end{tabular}


$\mathrm{V}$ : « c'est qu'entre eux quand même en général nos jeunes de quatorze quinze ans, ils ne parlent pas le russe et donc entre eux ils seront quand même obligés de parler l'anglais, ça sera au moins ça » préciser la référence. Le référent désigné par la construction détachée a été mentionné dans l'énoncé précédent, donc on peut présumer que sa répétition à l'aide d'un groupe nominal comporte d'autres fonctions, en plus de la référence simple, puisqu'il n'y a pas besoin de désambiguiisation :

\begin{tabular}{|l|l|l|l|l|l|l|}
\hline (7) & 1 & jasiis & need & ületalve & seisnud need & mädanenud \\
\hline & & et puis & DEM & durant_tout_l'hiver & rester-PPNDEM & pourrir-PPN \\
\hline
\end{tabular}

\begin{tabular}{|l|l|l|l|l|l|}
\hline & 2 & kartulid & neid & võeti & siis \\
\hline & pomme_de_terre. & pl DEM-PART & prendre-PRET.IMP & alors \\
\hline
\end{tabular}

\begin{tabular}{|l|l|l|l|l|l|}
\hline & 3 & ja & küpsetati ära siis see & oli & niiütelda \\
\hline & & et & cuire-PRET.IMPSPRTCL alors DEM & être-PRET.3sg & pour_ainsi_dire \\
\hline
\end{tabular}

\begin{tabular}{|l|l|l|l|l|l|}
\hline & 4 & see & niiöelda & lisa & veel \\
\hline & & DEM & pour_ainsi_dire & supplément & encore \\
\hline
\end{tabular}

« et puis ces pommes de terre de l'année d'avant, ces pommes de terre pourries, on les a donc prises et cuites et c'était pour ainsi dire encore un supplément. »

La reprise par un groupe nominal semble donner plus de poids à la description et permet aussi d'introduire un autre qualificatif, mädanenud "pourries ", ajouté après l'interruption de la construction initiale. Ce procédé contribue clairement au poids illustratif/argumentatif de l'énoncé et en comparaison des éléments lexicaux intégrés permet de mieux capter l'attention de l'interlocuteur. De manière générale, cette structure facilite le traitement de l'information en dissociant la description lexicale du référent et son intégration dans une relation prédicative, car l'élément détaché est relativement long et comporte aussi une réparation.

L'élément thématique dans l'exemple suivant see meil a été mentionné huit tours auparavant dans une construction présentative (ma näen=et teil on siin meili'aadress « je 
vois que vous avez ici une adresse e-mail »); ensuite, un autre sujet est introduit, après quoi le locuteur $\mathrm{V}$ reprend le référent mentionné auparavant (l'adresse e-mail du client) :

\begin{tabular}{|l|l|l|l|l|l|}
\hline$(8)$ & Kas & see & meil mis teil & siin on see & melesta punkt kitse punkt \\
\hline & Est-ce que & DEM & mail que vous-ADE & ici est DEM & melesta punkt kitse point \\
\hline
\end{tabular}

\begin{tabular}{|l|l|l|l|}
\hline & ri'äppl & mail & punktee- \\
\hline & ri'äppl & mail & pointee \\
\hline
\end{tabular}

\begin{tabular}{|l|l|l|l|l|}
\hline & ee kas & see & on & oige \\
\hline & est-ceque & DEM & est & correct \\
\hline
\end{tabular}

«Est-ce que ce mail que vous avez ici, ce melesta point kitse point ri’äppl mail point e e, est-ce qu'il est correct?»

Le constituant détaché se forme comme suit: d'abord le mot interrogatif kas est introduit et sera intégré dans la relation prédicative; l'élément détaché see meil est spécifié par une proposition relative mis teil siin on et ensuite, le locuteur épèle l'élément en question (l'adresse e-mail), en le faisant précéder par le démonstratif see. Après le groupe nominal complexe la construction initiale est suspendue et reprise avec une forme pronominale dans la proposition kas see on óige. Ce cas nous montre la possibilité qu'offre le détachement des éléments lexicaux de présenter au nominatif des constituants plus complexes en les plaçant au premier plan, ce qui rend leur interprétation plus aisée, surtout quand il s'agit d'éléments qui ressemblent aux citations ou labels et devraient par conséquent garder leur forme initiale, non modifiée.

\subsection{Gestion de la référence avec plusieurs référents concurrents}

Ici, nous observerons deux exemples dans lesquels un référent est réintroduit après que d'autres éléments ont été mentionnés entre-temps, ce qui ne permet pas d'utiliser un pronom pour y référer.

Dans le premier exemple (9), deux options sont d'abord proposées à un client qui s'informe par téléphone sur les horaires de bus. Le client demande la confirmation sur la deuxième option, à la suite de quoi l'agent propose encore une troisième option :

\begin{tabular}{|l|l|l|l|l|l|l|l|l|}
\hline (9) & 1 & H:. hh et kas & kella & 'viie & aeg & kuskil & 'läheb & Tartust \\
\hline & & que est-ce que & heure-GEN & cinq-GEN & temps & environ & aller-3sg & Tartu-ELA \\
\hline
\end{tabular}

\begin{tabular}{|l|l|l|l|}
\hline & 2 & 'Elvasse & 'buss. \\
\hline & & Elva-ILL & bus \\
\hline
\end{tabular}




\begin{tabular}{|l|l|l|l|l|l|l|l|l|}
\hline & 3 & V: & 'kuusteist & 'viiskümend & 'iga= päev, & 'seitseteist & 'kakskümend (.) & 'tööpäeviti. \\
\hline & & seize & cinquante & chaque jour & dix-sept & vingt & jours_ouvrables \\
\hline
\end{tabular}

\begin{tabular}{|c|c|c|c|c|c|}
\hline 4 & $\mathrm{H}:$ & seitseteist & 'kakskümend & tööpäeviti & jah? \\
\hline & & dix-sept & vingt & jours_ouvrables & oui \\
\hline
\end{tabular}

\begin{tabular}{|c|c|c|c|c|c|}
\hline 5 & V: jaa, & ja siis on & 'seitseteist & 'kakskümend=viis 'iga= & päev. \\
\hline & oui & et puis est & dix-sept & vingt-cinq & chaque jour \\
\hline
\end{tabular}

\begin{tabular}{|c|c|c|c|c|c|c|c|}
\hline 5 & $\begin{array}{ll}\text { H: } & \mathrm{mhmh} \\
\text {.hhhhhh } & \end{array}$ & aga kas & te & oskate & öelda & et & näiteks \\
\hline & mais & est-ce que & vous & savoir-2pl & dire-INF2 & que & exemple-TRL \\
\hline
\end{tabular}

\begin{tabular}{|c|c|c|c|}
\hline 6 & see & ee seitseteist kakskümmend & $\begin{array}{lll}\text { see } & \text { buss } & (0.3) \\
\text {.hhh } & & \end{array}$ \\
\hline & DEM & dix-sept vingt & DEM bus \\
\hline
\end{tabular}

\begin{tabular}{|c|c|c|c|c|c|}
\hline 7 & et $=e e$ & mis 'kell & ta on siin üleval & selles & Aardla peatuses. \\
\hline & que & quelle heure & il être-3sg ici & en_haut DEM-INE & Aardla arrêt-INE. \\
\hline
\end{tabular}

$\mathrm{H}$ : « est-ce que vers cinq heures environ il y a un bus qui va de Tartu à Elva ?» $\mathrm{V}$ : «seize heures cinquante tous les jours, dix-sept heures vingt les jours ouvrables. »

$\mathrm{H}:$ « dix-sept heures vingt les jours ouvrables, c'est cela?»

$\mathrm{V}:$ « oui, et puis il y a dix-sept heures vingt tous les jours. »

$\mathrm{H}$ : « mais mais pourriez-vous me dire, par exemple ce bus, celui à dix-sept heures vingt, ce bus, à quelle heure est-il ici, en haut, à l'arrêt Aardla? »

Après l'énoncé introductif (aga kas te oskate öelda "sauriez-vous dire»), le groupe nominal détaché (seitseteist kakskümmend see buss « dix-sept heures vingt ce bus») est précédé à la ligne 5 d'un élément pré-thématique typique, näiteks "par exemple » de sorte que l'on retrouve ici une caractéristique essentielle de la langue parlée spontanée, à savoir le fait de donner l'information par portions, séparées par des particules énonciatives ou d'autres moyens de formulation.

La transition entre l'élément détaché et la proposition principale est clairement marquée (pause, hésitation, répétition du subordonnant et qui introduit le complément du verbe öelda « dire » (kas te oskate öelda et näiteks)). 
41 pronom pour référer à celui qui l'intéresse, mais il recourt à une construction qui reprend l'élément nominal dans sa totalité, en lui juxtaposant l'élément lexical see buss « ce bus ». L'introduction relativement longue qui le précède est probablement due au fait que même si la mention précédente du référent n'est pas très loin (2 tours), une formulation plus longue est requise à cause de la similitude des deux référents mentionnés. l'expression d'un contraste implicite ou explicite. L'exemple suivant (10) contient une question alternative dans la proposition principale. Ici également, le référent désigné par le groupe nominal net Kanaari saared ("les îles Canaries») est introduit en contraste par rapport à un autre référent (l'Égypte) dont on a parlé dans le contexte précédent : le premier référent ayant été mentionné une fois dans une énumération au début de la conversation et après avoir discuté sur d'autres options, le locuteur y revient de nouveau :

\begin{tabular}{|l|l|l|l|l|l|}
\hline (10) & 1 & tundub & aga & näiteks & $\begin{array}{l}\text { net=ee } \\
\text { saared }\end{array}$ \\
\hline & Sembler-3sg & en_revanche & $\begin{array}{l}\text { exemple-TRL } \\
\text { DEM.pl }\end{array}$ & îles_Canaries \\
\hline
\end{tabular}

\begin{tabular}{|l|l|l|l|l|l|l|}
\hline & 2 & et kas & seal on & nagu & enamvähem & sama (.) \\
\hline & & PRTCL est-ce que & là être-3sg & comme & plus_ou_moins & même \\
\hline
\end{tabular}

\begin{tabular}{|c|c|c|c|c|c|}
\hline 3 & või kas & seal on & nagu & rohkem midagi & vaadata \\
\hline & ou est-ce que & là & être-3sg comme plus & quelque_chose & regarder-INF2 \\
\hline
\end{tabular}

\begin{tabular}{|l|l|l|}
\hline & 4 & või et noh. \\
\hline & & ou PRTCL PRTCL \\
\hline
\end{tabular}

«il semble en revanche que par exemple ces îles Canaries, que là-bas c'est plus ou moins la même chose ou est-ce qu'il y a plus de choses à voir là-bas ou alors? »

Après être réintroduit, ce référent est maintenu dans la discussion durant plusieurs tours, pendant que le locuteur $\mathrm{V}$ explique ses avantages.

Dans l'énoncé introductif, le verbe modal tunduma « paraître » est combiné avec deux particules qui s'utilisent fréquemment avant le nouveau thème, aga "en revanche " marquant une opposition et näiteks «par exemple » indiquant un choix à faire parmi une série de référents, comme dans l'exemple précédent. 
Le locuteur commence par énoncer quelque chose qui semble probable sur l'échelle épistémique, en employant le verbe tundub «il semble ", mais continue en posant une question composée d'alternatives coordonnées, introduites par kas (et kas seal on/või kas seal on) qui met en doute l'affirmation précédente. Il est possible que le locuteur ait aussi remarqué cette contradiction, car à la fin il essaie de formuler une troisième possibilité, avec une hésitation marquée par deux particules qui n'aboutit pas à un énoncé complet (või et noh).

L'élément détaché contient un marqueur d'hésitation ee après le démonstratif pluriel net ; ce type d'occurrence semble assez fréquent dans le cas des référents qui ne sont pas immédiatement présents. À part la recherche lexicale, cette construction peut être associée également au statut du référent: puisque le démonstratif est énoncé avant l'hésitation, le locuteur a déjà fait son choix dans la série paradigmatique des référents possibles et de ce fait cette hésitation semble indiquer davantage le statut informationnel du référent.

\subsection{Structuration du discours}

Les exemples suivants illustrent quelques cas où le détachement initial intervient soit pour clôturer une séquence, soit pour marquer le début d'une nouvelle séquence.

L'exemple (11) présente la fin d'une séquence plutôt monologique et fonctionne par conséquent au niveau du discours, permettant de clôturer un développement plus long où l'idée ou l'entité détachée peut déjà avoir été mentionnée ou encore représente le résultat de la réflexion du locuteur durant la séquence. Le fait d'arriver à une conclusion est indiqué par l'usage de la particule nii et :

\begin{tabular}{|l|l|l|l|l|l|}
\hline$(11)$ & 1 & sellepärast et (1.0) & ma: & ei tea & vanasti see \\
\hline & & parce_que & je & NEG savoir-NEG.1sg & autrefois DEM \\
\hline
\end{tabular}

\begin{tabular}{|c|c|c|}
\hline 2 & algas ikka & väga sügavalt \\
\hline & commencer-PRET.3sg PRTCL & très profondément \\
\hline
\end{tabular}

\begin{tabular}{|l|l|l|l|l|}
\hline & 3 & kodust $()$. & kodust & pihta vanaema vanaisa nende \\
\hline & & maison-ELA & maison-ELA ADV & grand-mère grand-père ils-GEN \\
\hline
\end{tabular}

\begin{tabular}{|l|l|l|l|l|}
\hline & 4 & suhtumine & nad niimoodi ei & õpetanud \\
\hline & & attitude & ils comme_ça NEG & enseigner-NEG.PRET.3pl \\
\hline
\end{tabular}

\begin{tabular}{|c|c|c|c|c|}
\hline 5 & ega ei & pidand & loenguid & vaid lihtsalt nad \\
\hline & ni NEG & tenir-NEG.PRET.3pl & leçon.pl-PART & mais simplement ils \\
\hline
\end{tabular}




\begin{tabular}{|l|l|l|l|l|}
\hline & 6 & 'olid & 'niisugused 'nagu & nad 'olid (.) \\
\hline & & être-PRET.3pl tels & comme ils & être-PRET.3pl \\
\hline
\end{tabular}

\begin{tabular}{|c|c|c|c|}
\hline 7 & nii et see & põhiline (.) & põhiline \\
\hline & donc DEM & principal & principal honnêteté \\
\hline
\end{tabular}

\begin{tabular}{|c|c|c|c|c|c|c|}
\hline 8 & see & tuli & ikkagi & suurelt & osalt s & ealt \\
\hline & DEM & venir-PRET.3sg & PRTCL+clitique & grand-ABL & partie-ABL & là-ABL \\
\hline
\end{tabular}

"parce que je ne sais pas, autrefois ça venait quand même de la maison, grandmère, grand-père, leur attitude, ils n'enseignaient pas, ils ne donnaient pas de leçons comme ça, mais tout simplement ils étaient tels qu'ils étaient, donc cette honnêteté, principalement, elle venait quand même de là. "

Dans ce qui précède, le locuteur a déploré la crise actuelle de l'éthique, en donnant quelques exemples par une juxtaposition des éléments de son argumentation aux lignes 4 et 5 (vanaema vanaisa nende suhtumine "grand-mère grand-père leur attitude "). Le locuteur parle en faisant des pauses et en accentuant chaque mot séparément, ce qui contribue à l'impression générale d'un discours pédagogique, avec un engagement personnel assez important.

51 Il s'agit ici d'un cas où une construction détachée est utilisée dans un extrait monologique et fonctionne clairement au niveau du discours: dans ce type d'occurrence, l'élément détaché se rapporte à une entité/idée (abstraite) qui a pu déjà être mentionnée dans le discours précédent ; l'énoncé avec l'élément détaché en tête présente le résultat du développement de cette idée.

La conclusion du passage qui contient aussi l'élément détaché commence par la combinaison de deux particules nii et (ligne 8). Ici la construction détachée ne semble pas relever d'un contraste interne ou de l'extraction d'une entité parmi un groupe d'éléments. Dans les passages précédents le locuteur a déploré la crise actuelle de la morale qui, dans une certaine mesure, peut constituer un contraste avec l'idée principale de la séquence en question. Ainsi, la construction détachée contribue à la structuration générale de la séquence.

Il faut également noter l'organisation de la proposition qui introduit les référents humains dont il s'agit dans ce passage (vanaema vanaisa nende suhtumine): elle est caractérisée par une juxtaposition d'éléments typique de la langue orale. Ici cela pourrait contribuer à souligner le message du locuteur, puisque les deux personnes mentionnées comme exemples constituent les éléments centraux de son argumentation et le locuteur y réfère plus tard à l'aide de pronoms personnels.

L'exemple suivant (12) montre un autre cas où un énoncé avec un élément détaché en tête a pour fonction de conclure un passage. Le référent désigné par le GN see haridus poistel a été développé bien longtemps avant cette occurrence (plus de 60 tours de parole avant) et le retour à ce référent sert à clore la discussion : 


\begin{tabular}{|l|l|l|l|l|l|}
\hline$(12)$ & H: & aga: mis sa arvad & kas: & nagu need sinu & 'kasvatusmeetodid .hh \\
\hline & & mais que tu penser.2sg & est-ce que & comme DEM & tes méthodes_d'éducation \\
\hline
\end{tabular}

\begin{tabular}{|l|l|l|l|}
\hline & 2 & nagu 'mõjusid $=e t$ & kas 'kasvasid nendest \\
\hline & comme agir-PRET.3pl PRTCL & est-ce que grandir-PRET.3pl DEM-ELA \\
\hline
\end{tabular}

\begin{tabular}{|l|l|l|l|l|l|}
\hline & 3 & 'lastest (.)sellised lapsed & nagu & sa & 'ootasid. \\
\hline & & enfant.pl-ELA tel.pl enfant.pl & comme & tu & attendre-PRET.2sg \\
\hline
\end{tabular}

\begin{tabular}{|l|l|l|l|l|}
\hline & 4 & V: & .hh ma ütleksin & jaa, enamvähem. \\
\hline & & & je dire-COND.1sg & oui plus_ou_moins \\
\hline
\end{tabular}

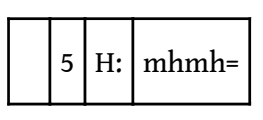

\begin{tabular}{|l|l|l|l|l|l|l|l|}
\hline & 6 & V: & $\begin{array}{l}\text { =võibolla jah, } \\
. \text { hh }\end{array}$ & midagi & jäi & seal (.) & muidugi \\
\hline & & peut-être oui & quelque_chose & rester-PRET.3sg & là & bien_sûr & \\
\hline
\end{tabular}

\begin{tabular}{|l|l|l|l|l|l|l|l|l|}
\hline & 7 & see.$h h$ & 'haridus & poistel, & sellest & on & mul & väga 'kahju \\
\hline & DEM & éducation & garçon.pl-ADE & DEM-ELA & être.3sg & je-ADE & très dommage \\
\hline
\end{tabular}

$\mathrm{H}$ : «Mais penses-tu que tes méthodes éducatives ont été efficaces, est-ce que tes enfants sont devenus tels que tu avais espéré ?»

$\mathrm{V}:$ « Je dirais que oui, plus ou moins.»

$\mathrm{H}:$ : Mhmh »

$\mathrm{V}$ : «Peut-être quelque chose est quand même resté, bien sûr, l'éducation des garçons, ça je trouve très dommage. »

La construction détachée vient à la fin de la réponse que le locuteur $\mathrm{V}$ donne à la question de $\mathrm{H}$. La réponse est quelque peu hésitante avant l'introduction du groupe nominal see haridus poistel « l'éducation des garçons » (ligne 7); ce dernier est précédé la particule muidugi « bien sûr » qui réfère à leur connaissance partagée : la position de la locutrice avait été exprimée plus tôt dans la conversation. Ici, la construction avec un élément détaché en tête permet d'énoncer à la fin d'une séquence une idée claire au sujet d'un référent qui a été mentionné auparavant. Ainsi, elle contribue à donner une cohérence interne à tout un passage de discours. Dans cet exemple, comme dans plusieurs autres extraits (exemples 6,10), la construction détachée vient résoudre une séquence d'hésitations et l'on peut suggérer que par rapport à un GN intégré dans la proposition principale, le détachement suivi de la reprise pronominale permet d'introduire un élément dans un discours construit en temps réel avec moins de charge 
informationnelle. Dans ces cas-là, il peut s'agir de la recherche lexicale qui aboutit à un élément présenté au nominatif, mais l'utilisation de ce procédé peut être indissociable de l'intention du locuteur qui veut souligner un aspect de son argumentation.

Les deux extraits suivants permettront de discuter les occurrences où l'élément détaché introduit un référent qui sera maintenu dans le discours durant de nombreux énoncés, repris par des pronoms anaphoriques. Les relations référentielles deviennent plus complexes lorsqu'il y a plusieurs référents traités à tour de rôle, comme dans le premier exemple :

\begin{tabular}{|l|l|l|l|l|l|l|l|}
\hline$(13)$ & 1 & H: & [meil & oli ee (.) & meil & oli ee] (1.0) & meil \\
\hline & & [nous-ADE & être-PRET.3sg & nous-ADE & être-PRET.3sg] & nous-ADE & \\
\hline
\end{tabular}

\begin{tabular}{|c|c|c|c|c|c|c|}
\hline 2 & oli ee $(0.8)$ & vanasti: & poisid & 'väiksed & olid, & sis \\
\hline & être-PRET.3sg & autrefois $\mathrm{g}$ & arçon.pl & petit.pl & être-PRET.3pl & alors \\
\hline
\end{tabular}

\begin{tabular}{|c|c|c|c|c|}
\hline 3 & oli & meil & üks 'oinas & kodus. \\
\hline & être-PRET.3sg & nous-ADE & un bélier_castré & maison-INE \\
\hline
\end{tabular}

\begin{tabular}{|c|c|c|c|c|c|c|}
\hline 4 & (0.8) noh & ja & nemad & 'õpetasid & kurjad & vaimud \\
\hline & PRTCL & et & ils & enseigner-PRET.3pl & méchant. & pl esprit.pl \\
\hline
\end{tabular}

\begin{tabular}{|l|l|l|l|}
\hline & 5 & selle oina & nii ‘kurja(h) ks hehe \\
\hline & DEM-GEN bélier-GEN si & méchant-TRL \\
\hline
\end{tabular}

\begin{tabular}{|l|l|l|l|l|l|l|}
\hline & 6 & et & 'Juuli-tädi $(0.5)$ see ei & tohtind & ültse & liikuda \\
\hline & & que & Juuli-tante DEM NEG & pouvoir-PRET.3sg & du_tout & bouger-INF2 \\
\hline
\end{tabular}

\begin{tabular}{|l|l|l|l|l|l|l|}
\hline & 7 & niigu & 'kummardas & nii & oinas & pani ‘plaksti:, \\
\hline & & dès_que & se_baisser-PRET.3sg & voilà & bélier & mettre-PRET.3sg ONOM \\
\hline
\end{tabular}

\begin{tabular}{|c|c|c|c|c|c|}
\hline 8 & ja & ta 'käis & 'lahtiselt ka & ja siis & pärast panime \\
\hline & et il & Aller-PRET.3sg & détaché aussi & et puis après & mettre-PRET.3sg \\
\hline
\end{tabular}

\begin{tabular}{|c|c|c|c|c|c|c|c|}
\hline 9 & küll (0.5) & 'köide & see & läks & ügsgord & 'põllu= & pääle \\
\hline
\end{tabular}




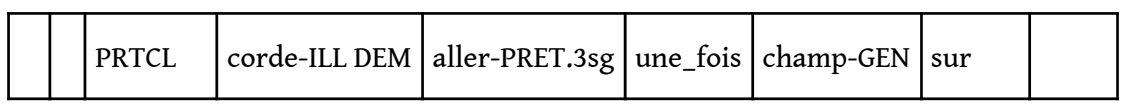

\begin{tabular}{|l|l|l|l|l|l|l|}
\hline & 10 & teda & ‘edasi & lööma & näed & 'niivisi, \\
\hline & & il-PART & plus_loin & frapper-SUP & voir.2sg & comme ça \\
\hline
\end{tabular}

\begin{tabular}{|l|l|l|l|l|l|}
\hline & 11 & $(0.5)$ & jajjäi & sinna kummargi jäi & sinna \\
\hline & & & et rester-PRET.3sg là & accroupi rester-PRET.3sg & là \\
\hline
\end{tabular}

\begin{tabular}{|l|l|l|l|l|}
\hline & 12 & oina & 'meelevalda & ja \\
\hline & & bélier-GEN & merci-ILL & et \\
\hline
\end{tabular}

\begin{tabular}{|l|l|l|l|l|l|}
\hline & 13 & ei saand ‘ära & ka sealt & enam. & niigu \\
\hline & & NEG pouvoir-NEG.PRET.3sg PRTCL & aussi là-ABL & plus & dès_que \\
\hline
\end{tabular}

\begin{tabular}{|l|l|l|l|l|l|l|}
\hline & 14 & niigu & 'tõusis & nii oinas & pani & jälle \\
\hline & & dès_que & se_lever-PRET.3sg & que bélier & mettre-PRET.3sg & de_nouveau \\
\hline
\end{tabular}

\begin{tabular}{|l|l|l|l|l|l|l|l|l|}
\hline & 15 & plaks ‘pikali. & hehe ja siis, & $\mathrm{ku}$ & 'ära & tapsime, & (1.2) ei & 'poisid \\
\hline & ONOM & couché & et puis & quand PRTCL & tuer-PRET.1pl & non & garçon.pl \\
\hline
\end{tabular}

\begin{tabular}{|l|l|l|l|l|l|l|}
\hline & 16 & noh & nemad ei & 'söö & seda: (0.5) & ee seda \\
\hline & & PRTCL & ils & NEG manger-NEG.3pl & DEM-PART & DEM-PART \\
\hline
\end{tabular}

\begin{tabular}{|l|l|l|l|l|}
\hline & 17 & just see & ma=i & 'mäleta \\
\hline & & justement DEM & je NEG & rappeler.NEG.1sg \\
\hline
\end{tabular}

\begin{tabular}{|l|l|l|l|l|l|}
\hline & 18 & mis ta 'nimi & oli & [sel & 'oinal.] (.) \\
\hline & & quel son nom & être-PRET.3sg & DEM-ADE & bélier-ADE \\
\hline
\end{tabular}

« Nous avions autrefois quand les garçons étaient petits, nous avions un bélier castré à la maison et donc ces coquins ont rendu ce bélier si méchant que la tante Juuli ne pouvait pas bouger du tout. Dès qu'elle se baissait, le bélier lui donnait un coup. Il n'était pas attaché non plus, plus tard on l'avait attaché. Et elle est partie sur le champ pour avancer son piquet, tu vois comme ça et elle est restée là-bas 
accroupie à la merci du bélier et ne pouvait plus s'en aller : dès qu'elle se levait, le bélier la renversait encore. Et quand on l'a tué - non, les garçons, eux n'en mangent pas je ne me souviens pas quel était son nom, ce bélier. » deuxième mention lexicale de l'animal (oinas), le pronom personnel ta reste " assigné » à cet élément (lignes 8 et 10), tandis que le démonstratif see sera employé pour référer à l'être humain (see läks ügsgord pôllu=pääle teda edasi lööma). Ce schéma peu ordinaire peut être expliqué par le fait que l'animal semble avoir un rôle plus central dans cette séquence (mentionné huit fois, y compris deux références anaphoriques et une anaphore zéro) et que cette stratégie permet de poursuivre la narration sans devoir réintroduire l'élément lexical.

Cet extrait contient deux cas de détachement initial : d'abord, le premier référent est introduit par un nom propre (Juuli-tädi) à la ligne 6 et repris avec le pronom see, puis on trouve un deuxième détachement initial à la fin de la séquence ei poisid noh nemad ei söö seda (lignes 15 et 16). Dans les deux exemples, l'élément détaché est séparé de la proposition principale par un moyen linguistique ou paralinguistique : par une pause dans le premier cas et par la particule noh dans le deuxième cas.

animaux élevés dans la ferme familiale ; la séquence en question comporte en outre une longue description du mauvais caractère de l'animal présenté comme exemple, un bélier castré.

référentielles dans cet extrait.

dans une expression cadrative à valeur temporelle vanasti poisid väiksed olid « autrefois quand les garçons étaient petits », ensuite le locuteur y réfère par le pronom nemad « ils» (ligne 4); puis, un nouveau référent humain, Juuli-tädi, est introduit dans une construction détachée, à laquelle on fait d'abord référence par le démonstratif see (ligne 6), ensuite par l'anaphore zéro (ligne 7). dans le temps et d'autre part par le fait que l'entité humaine principale de cet extrait est poisid «les garçons"; une autre particularité qui peut influencer l'usage (et le contraste) des pronoms utilisés est le fait que le locuteur décrit de manière détaillée l'interaction entre un humain et un animal. Puisque le référent introduit est nouveau dans le texte, la construction détachée (Juuli-tädi) permet de présenter et d'assimiler cette information graduellement.

Quant aux relations référentielles dans toute la séquence, on peut constater qu'après la Le référent nommé poisid est repris à la fin de la séquence dans une construction détachée servant à introduire un énoncé qui résume l'attitude des garçons telle qu'elle était perçue par le locuteur (la particule de négation ei rapporte le refus exprimé par les garçons). À l'intersection de l'élément détaché et de la proposition principale se trouve la particule noh dont la fonction peut également être liée à la composition polyphonique de cet énoncé : en effet, ce dernier se distingue du reste de la séquence qui est une narration au passé - par l'utilisation du verbe sööma « manger » au présent. Ce changement de perspective indique qu'il s'agit ici des paroles rapportées des garçons. Le locuteur a l'intention de poursuivre son énoncé, en essayant de se rappeler le nom du bélier - la répétition du pronom seda marque cet effort de remémoration. 
Du point de vue de l'information, le référent désigné par le nom poisid est l'un des trois principaux acteurs de cet extrait, mais d'abord il sert à poser le cadre temporel de la séquence "quand les garçons étaient petits " et ce n'est que plus tard à la fin de l'extrait, que le locuteur réintroduit ce référent par une construction détachée.

L'exemple suivant se caractérise également par une longue séquence anaphorique: l'élément lexical introduit au début de l'extrait a un sens générique (inimene "une personne »), car le locuteur parle des acheteurs typiques dans une librairie où elle travaillait, mais par la suite ce référent est maintenu pendant longtemps avec une forme ta « il ». Il est question de ce référent déjà dans les tours précédents où le locuteur y réfère par le substantif pluriel inimesed « les gens ». Le passage du pluriel au singulier se fait à l'aide d'une construction détachée au début de l'extrait ci-dessous :
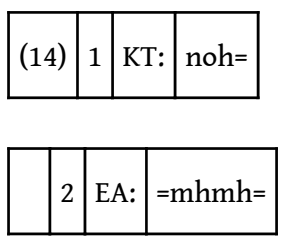

\begin{tabular}{|l|l|l|l|l|l|l|l|l|}
\hline & 3 & KT:= & 'inimene noh> & hea'küll & ma ei & ‘lähe & teda & 'segama \$ \\
\hline & & Personne PRTCL & PRTCL & je NEG & aller-NEG.1sg & il-PART & déranger-SUP \\
\hline
\end{tabular}

\begin{tabular}{|l|l|l|l|l|l|l|l|l|}
\hline & 4 & siis kui & Ta juba & loeb & ega & ma ei & saa & teda \\
\hline & alors quand & il déjà & lire-3sg & NEG & je NEG & pouvoir-NEG.1sg & il-PART \\
\hline
\end{tabular}

\begin{tabular}{|c|c|c|c|c|c|c|}
\hline 5 & aidata & ‘lugeda & eks $<\$$ hee & aga noh(.) & nii & ‘alguses \\
\hline & aider-INF2 & lire-INF2 & n'est-ce_pas & Mais PRTCL & comme & début-INE \\
\hline
\end{tabular}

\begin{tabular}{|l|l|l|l|l|l|l|}
\hline & 6 & kui & ta nagu & 'otsib $=$ või(.)>a & ta onise & 'segaduses \\
\hline & & quand & il comme & chercher-3sgou & il estlui-même & confusion-INE \\
\hline
\end{tabular}

\begin{tabular}{|c|c|c|c|c|c|c|c|}
\hline 7 & alles & ta ei & tea & ka & ‘täpselt & 'mida & ta nagu \\
\hline & encore & Il NEG & savoir.NEG.3sg & non_plus & exactement & ce_que & il comme \\
\hline
\end{tabular}

\begin{tabular}{|c|c|c|c|c|c|c|}
\hline 8 & 'tahab= & $\mathrm{ja}<(-)$ & ta tahab & alles & mingit & 'pilti \\
\hline & vouloir-3sg & et & il vouloir-3sg & encore & quelque-PART & image-PART \\
\hline
\end{tabular}

\begin{tabular}{|c|c|c|c|}
\hline & luua & $j a=j a$ & üldse ja = noh \\
\hline
\end{tabular}




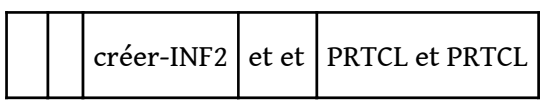

KT: «Eh ben quelqu'un, bon, je ne vais pas le déranger quand il lit déjà, je ne peux pas l'aider à lire, n'est-ce pas, mais bon, au début quand il cherche ou est encore incertain, il ne sait pas encore exactement ce qu'il veut et qu'il a envie de se faire une idée et que voilà »

L'élément détaché est repris par le pronom ta ou teda (la forme partitive de ta). On peut relever également deux particules qui se placent après l'élément lexical à la ligne 3 : noh, hea küll. La particule noh peut être utilisée pendant la recherche du terme adéquat et elle fonctionne aussi comme marqueur de segmentation à l'intersection des séquences (dans cet exemple en combinaison avec aga à la ligne 5). Hea küll est un marqueur de concession qui se combine avec noh à l'intersection de l'élément détaché et la prédication qui suit.

Le pronom ta sert à référer au référent non spécifique durant tout le développement de l'exemple par le locuteur KT, mais plus loin dans la conversation, le référent sera réintroduit par le nom pluriel (inimesed). Il est à noter que le terme détaché n'est pas déterminé par le démonstratif see, contrairement aux autres exemples : cette omission permet justement d'en faire une lecture générique.

\section{Conclusion}

Les constructions comportant un détachement initial semblent avoir dans le discours plusieurs fonctions. D'abord, il convient de souligner le fait que les caractéristiques formelles de cette structure permettent de présenter une entité complexe ou longue au nominatif, suivie par une reprise pronominale éventuellement à un autre cas dans la proposition principale. Dans ces cas-là, on trouve des éléments de type «citation » ou autres, tels que les adresses, les noms propres, les groupes nominaux complexes, etc. La présentation au nominatif (d'une entité parfois très longue dans le cas des propositions relatives) allège la charge informationnelle et offre également la possibilité au locuteur de corriger la référence (accord, recherche lexicale, parfois généralisation). Souvent, la construction détachée est précédée de différents éléments qui marquent une difficulté de formulation: ainsi, la construction détachée représente une étape dans la construction du discours, permettant au locuteur de se focaliser ensuite sur le rhème qui sera exprimé avec la rection appropriée.

69 La construction avec un élément détaché est employée aussi bien pour ouvrir une séquence en introduisant un nouvel élément dans le discours que pour clôturer une séquence. Dans le premier cas, le lien avec le cadre référentiel est moins marqué l'élément introduit est mentionné pour la première fois dans la conversation, soit il est présenté comme étant connu par les interlocuteurs, soit il peut être associé au cadre thématique de la conversation au sens large.

Dans presque tous les cas, les éléments détachés portent le marqueur d'identifiabilité see/need, ce qui indique entre autres leur statut informationnel. On peut globalement distinguer deux types d'occurrences :

1. Les référents présents dans la situation ou le contexte, mentionnés explicitement, parfois aussi réintroduits (en contraste ou retour à un sujet précédent). 
2. Focalisation sur un ou plusieurs membres extraits d'un groupe, présentés pour illustrer d'une argumentation, c'est-à-dire que le référent est présent dans le cadre référentiel. Plus rarement on trouve dans cette position des éléments mentionnés pour la première fois qui ne semblent pas avoir de rapport avec le discours précédent, mais qui sont néanmoins présentés par le locuteur comme potentiellement identifiables : cela est confirmé aussi par le fait que dans ces cas-là les autres participants n'interviennent pas pour préciser la référence - soit le référent est considéré comme un exemple accessoire qui ne demande pas d'identification précise, soit il est associé au discours par ce qui en est dit par la suite.

71 Les exemples analysés montrent qu'en général, le détachement initial en estonien ne sert pas à introduire de nouveaux thèmes, des référents « inaccessibles ».

Un autre aspect encore peu exploré concerne les effets stylistiques ou rhétoriques de ces constructions. De par leur forme et la diversité des relations référentielles que l'on peut trouver associées à ces structures, on peut affirmer que les détachements initiaux dans le discours aident à capter l'attention des participants, à structurer ou à ponctuer le discours qui est en train de se construire en temps réel et à souligner l'engagement personnel du locuteur.

\section{Liste d'abréviations}

\begin{tabular}{|l|l|}
\hline Abréviation & Signification \\
\hline ABE & abessif \\
\hline ABL & ablatif \\
\hline ADE & adessif \\
\hline ADV & adverbe \\
\hline AUX & auxiliaire \\
\hline COM & comitatif \\
\hline COND & conditionnel \\
\hline DEM & démonstratif \\
\hline ELA & élatif \\
\hline GEN & génitif \\
\hline ILL & illatif \\
\hline IMP & impératif \\
\hline IMPS & impersonnel \\
\hline INE & inessif \\
\hline INF2 & infinitif en -da \\
\hline
\end{tabular}




\begin{tabular}{|l|l|}
\hline NEG & forme négative \\
\hline ONOM & onomatopée \\
\hline PART & partitif \\
\hline pl & pluriel \\
\hline PPN & participe passé en -nud \\
\hline PPT & participe passé en -tud \\
\hline PRET & prétérit \\
\hline PRTCL & particule \\
\hline Sg & singulier \\
\hline SUP & supine \\
\hline TRL & translatif \\
\hline 1 & $1^{\text {ère personne }}$ \\
\hline 2 & $2^{\text {ème personne }}$ \\
\hline 3 & $3^{\text {ème personne }}$ \\
\hline
\end{tabular}

\section{Conventions de transcription}

\begin{tabular}{|l|l|}
\hline$[\ldots]$ & énoncés en chevauchement \\
\hline$()$. & micropause (moins de $0.5 \mathrm{sec})$ \\
\hline$(0.6)$ & durée de la pause \\
\hline i $:$ & extension du son \\
\hline. & mot ou partie du mot accentué \\
\hline$=$ & énoncés continus \\
\hline$<\ldots>$ & ralentissement du rythme \\
\hline$>\ldots<$ & accélération du rythme \\
\hline$\$ \ldots \$$ & passage prononcé en riant \\
\hline.$h \mathrm{~h}$ & inspiration audible \\
\hline hh & expiration audible \\
\hline
\end{tabular}




\begin{tabular}{|l|l|}
\hline$\cdot$ & intonation descendante \\
\hline$\{-\}$ & passage inaudible \\
\hline- & mot tronqué \\
\hline
\end{tabular}

\section{BIBLIOGRAPHIE}

\section{Références}

ASHBY William J., 1988, “The syntax, pragmatics and sociolinguistics of left and right dislocation in French", Lingua ${ }^{\circ} 75$, p. 203-229.

BALLY Charles, 1944 [1932], Linguistique générale et linguistique francaise, $2^{\text {nd }}$ édition entièrement refondue, Berne : A. Francke S.A.

COMBETTES Bernard, 1983, Pour une grammaire textuelle, Bruxelles : De Boeck.

COMBETTES Bernard, 1998, Les constructions détachées en français, Paris : Ophrys.

COMBETTES Bernard, 1999, «Thématisation et topicalisation : leur rôle respectif dans l'évolution du français ", in GUIMIER Claude, La thématisation dans les langues. Actes du colloque de Caen, 9-11 octobre 1997, Berne : Peter Lang, p. 231-245.

COMBETTES Bernard, NEVEU Franck, Les linguistiques du détachement. Actes du Colloque International de Nancy (7-9 juin 2006), Berne : Peter Lang.

ENKVIST Nils Erik, 1984, “Contrastive linguistics and text linguistics”, in FISIAK Jacek, Contrastive linguistics: prospects and problems, The Hague: Mouton, p. 45-67.

ENKVIST Nils Erik, 1987, “Text linguistics for the applier: an orientation”, in CONNOR Ulla et KAPLAN Robert B., Writing across languages: analysis of L2 text, Reading, MA: Addison-Wesley, p. 23-42.

ERELT Mati et al., 1993, Eesti keele grammatika II. Süntaks. Lisa: Kiri, Tallinn: Eesti Teaduste Akadeemia Eesti Keele ja Kirjanduse Instituut.

ETELÄMÄKI Marja, 2006, Toiminta ja tarkoite. Tutkimus suomen pronominista tämä, Helsinki: SKS.

FERNANDEZ-VEST Marie Madeleine Jocelyne, 2004, « Mnémème, antitopic : le post-rhème, de l'énoncé au texte » in CARTER-THOMAS Shirley et FERNANDEZ-VEST Marie Madeleine Jocelyne, Structure informationnelle et particules énonciatives, Paris : L'Harmattan, p. 65-104.

FERNANDEZ-VEST Marie Madeleine Jocelyne, 2006, « Vers une typologie linguistique du détachement à fondement ouralien d'Europe », Bulletin de la Société Linguistique de Paris, $n^{\circ} 101$ (1), p. 173-224. 
FERNANDEZ-VEST Marie Madeleine Jocelyne, 2009, « Vers une typologie informationnelle des constructions à détachement », in APOTHELOZ Denis, COMBETTES Bernard, NEVEU Frank, Les linguistiques du détachement ; actes du colloque international de Nancy (2006), Peter Lang, p. 251-262. GELUYKENS Ronald, 1992, From discourse process to grammatical construction: on left-dislocation in English, Amsterdam/Philadelphia: John Benjamins.

GUNDEL Jeannette 1988, “Universals of topic-comment structure", in HAMMOND Michael et al., Studies in Syntactic Typology, John Benjamins, p. 209-239.

GUNDEL Jeannette, 2012, "Pragmatics and information structure", in ALLAN Keith et JASZCZOLT Kasia, Cambridge Handbook of Pragmatics, p. 585-599.

GUNDEL Jeannette, HEDBERG Nancy, ZACHARSKI Ron, 1993, "Cognitive status and the form of referring expressions in discourse", Language, $\mathrm{n}^{\circ} 69$, p. 274-307.

HAKULINEN Auli et al., 2004, Iso suomen kielioppi. Helsinki: SKS.

HELASVUO Marja-Liisa, 2001, Syntax in the making. The emergence of syntactic units in Finnish conversation, Amsterdam: John Benjamins.

LAMBRECHT Knud, 1994, Information structure and sentence form. Topic, focus and the mental representations of the discourse referents, Cambridge: Cambridge University Press.

LI Charles N., THOMPSON Sandra, 1976, "Subject and topic. A new typology of language", in LI Charles N., Subject and topic, New York: Academic Press.

MARNETTE Sophie, 2005, Speech and thought presentation in French, Amsterdam and Philadelphia: John Benjamins.

MASLOVA Elena, BERNINI Giuliano, 2006, "Sentence topics in the languages of Europe and beyond", in BERNINI Giuliano et SCHWARTZ Marcia L., Pragmatic organization of discourse in the languages of Europe. Empirical approaches to language typology, 20-8, Berlin, New York: Mouton de Gruyter, p. 67-120.

MCLAUGHLIN Mairi, 2011, "When written is spoken: dislocation and the oral code", French Language Studies, $\mathrm{n}^{\circ} 21$, p. 209-229.

PRINCE Ellen, 1981, “Toward a taxonomy of given-new information”, in cole Peter, Radical pragmatics, New York: New York Academic Press, p. 223-255.

\section{Sources}

HARGLA Indrek, 2010, Oleviste mõistatus, Tallinn : Varrak. HARGLA Indrek, 2013, L'Enigme de Saint Olav : Melchior l'Apothicaire, traduit de l'estonien par Jean Pascal Ollivry, Montfort-en-Chalosse : Gaïa Éditions.

\section{NOTES}

1. Voir la liste des abréviations en fin d'article. 


\section{RÉSUMÉS}

L'article s'intéresse au fonctionnement discursif du détachement initial en estonien oral et analyse son rôle dans la construction et la structuration du discours en s'appuyant sur des exemples tirés de corpus oraux. Les constructions à détachement ont été considérées comme des universaux dans la langue parlée spontanée et le détachement initial est souvent associé au constituant informationnel thème.

Les référents des constructions à détachement peuvent être assez variés en ce qui concerne leur statut informationnel, mais généralement ils sont présents dans le discours ou dans le cadre thématique; il convient de les analyser au niveau du discours, puisque souvent ils sont maintenus dans la conversation pendant une séquence entière.

Un élément détaché au nominatif introduit en tête d'énoncé qui sera repris ensuite par un pronom dans la proposition principale présente un certain nombre de caractéristiques spécifiques, si l'on le compare à un GN intégré: en plus de réintroduire une entité déjà mentionnée ou présente dans le contexte, il permet d'introduire des éléments complexes, tels que adresses, noms propres, etc. avec une moindre charge informationnelle, et se trouve souvent au cœur du travail de formulation, puisqu'il apparaît fréquemment en association avec des réparations, difficultés de formulation et recherches lexicales.

Les constructions à détachement initial contribuent clairement à la structuration du discours audelà de l'énoncé, car elles sont employées aussi bien pour ouvrir que pour clôturer des séquences longues.

This article deals with initial detachments in spontaneous oral Estonian and analyses their role in the construction and structuration of the discourse, based on the analysis of examples from oral corpora. These constructions are considered as being universal in spontaneous oral language and the initial detachment has been often associated to the theme of the utterance.

Detachment constructions are able to encompass a wide range of referents with regards to their informational status in the discourse, but generally they are present in the conversation or in the thematic frame; as the referents of these constructions can be maintained in the conversation during a whole sequence, they should be considered at the level of the discourse.

A detached lexical element in nominative, followed by the resumptive pronoun in the main clause has, as a construction, some specific characteristics, compared to an integrated noun phrase: besides of the fact that it reintroduces an entity that has been already mentioned or that is present in the context, it allows to introduce complex elements by alleviating their informational charge, such as addresses, names etc., and it is often in the centre of the formulation work, as it appears frequently associated with repairs, difficulties of formulation and lexical retrieval.

The constructions with a detached lexical element contribute clearly to the structuration of the discourse beyond the single utterance, as they are used also for opening as well as resuming longer sequences.

Käesolevas artiklis käsitletakse algteemat sisaldavaid lahktarindeid spontaanses eesti suulises kõnes, analüüsides nende konstruktsioonide rolli diskursuse ülesehitamisel ja struktureerimisel. Niisuguseid tarindeid peetakse suulises spontaanses kõnes universaalseteks ning algteemat sisaldavad konstruktsioone on sageli seostatud infostruktuuri raamistikust pärit teema mõistega. Nende tarindite referentide informatsiooniline staatus võib olla mitmesugune, kuid enamasti on referendid kas mainitud eelnenud vestluses või on nad seotud üldise temaatilise raamistikuga; kuivõrd referent võib püsida kõnes pikema lõigu vältel, tuleks neid konstruktsioone vaadelda ka diskursuse tasandil. 
Võrreldes leksikaalselt integreeritud noomenifraasidega on ettetõstetud teemaga lahktarinditel mõningad spetsiifilised jooned: lisaks eelnevalt mainitud või kontekstist tuletatava referendi uuesti sissetoomisele võimaldavad need tarindid tuua info töötlemise seisukohast väiksema pingutusega sisse keerukamaid elemente nagu aadressid, nimed jne, ning lisaks sellele paiknevad need konstruktsioonid teksti loomise ja formuleerimise keskmes, kuna nendega seoses esineb mitmesuguseid parandusi ning formuleerimisraskusi, sealhulgas ka sobiva leksikaalse elemendi leidmisel.

Algteemat sisaldavad lahktarindid annavad selge panuse diskursuse struktureerimisse ka väljaspool ühe lausungi piiri, kuna neid kasutatakse nii pikemate lõikude sissejuhatamisel kui ka kokkuvõtmisel.

\section{INDEX}

Thèmes : linguistique

disciplines finnois, estonien

motscleset sõnade järjekord, tematiseerimine, info struktureerimine, kõnekeel

Mots-clés : ordre des mots, thématisation, structuration de l'information langue parlée

motsclesru ПОРЯДОК СЛОВ, ТЕМАТИЗАЦИЯ, ОФОРМЛЕНИЕ ВЫСКАЗЫВАНИЯ, УСТНАЯ РЕЧЬ

Keywords : Word Order, Topicalisation, Information Structure, Oral Language 